Review began $01 / 03 / 2022$ Review ended 01/18/2022 Published 01/21/2022

(๑) Copyright 2022

Turgut et al. This is an open access article distributed under the terms of the Creative Commons Attribution License CC-BY 4.0. which permits unrestricted use, distribution, and reproduction in any medium, provided the original author and source are credited.

\title{
Standard and Newly Defined Prognostic Factors Affecting Early Mortality After Hip Fractures
}

\author{
Necmettin Turgut ${ }^{1}$, Abdullah Meriç Ünal ${ }^{2}$ \\ 1. Orthopedics and Traumatology, Isparta City Hospital, Isparta, TUR 2. Orthopedics and Traumatology, Özel Meddem \\ Hospital, Isparta, TUR
}

Corresponding author: Necmettin Turgut, drnecmettinturgut@hotmail.com

\begin{abstract}
Purpose: Early mortality rate in geriatric patients after hip fractures remains very high. Determining the prognostic factors is crucial for decreasing early mortality. This study aimed to evaluate the prognostic risk factors affecting early mortality after hip fracture in the elderly.

Methods: Medical records of 335 patients with age 70 years or older who sustained hip fractures which were treated by hemiarthroplasty or proximal femoral nailing between May 2017 and May 2019 were reviewed. Neutrophil/lymphocyte ratio (NLR) and platelet/lymphocyte ratio (PLR) were investigated for validity as the new prognostic markers. The other variables included age, gender, type of surgery, type of implant, type of anesthesia, American Society of Anesthesiologists (ASA) score, presence of comorbidities, delirium, length of hospital stay, time delay to surgery, number of erythrocyte transfusions, and laboratory data were assessed for 30-day, 90-day, and one-year mortality. Univariate analysis and logistic regression analysis were used to determine the associated mortality.
\end{abstract}

Results: Thirty-day mortality rate was $10.4 \%$ and was associated with being aged $\geqslant 90$ years (p-value: 0.013 , odds ratio \{OR\}: 0.13 ) and ASA score of 4 (p-value: 0.019 , OR: 0.22 ). Ninety-day mortality rate was $21.5 \%$ and was associated with age (p-value: 0.002), being aged 80-89 years (p-value: 0.032, OR: 0.43), being aged $\geqslant 90$ years (p-value: 0.001 , OR: 0.13), general anesthesia (p-value: 0.016, OR: 0.41 ), preoperative high NLR level (p-value: 0.028, OR: 1.05), high blood urea nitrogen (BUN) level (p-value: 0.049, OR:1.02). One year mortality rate was $33.7 \%$ and independent significant prognostic risk factors were determined as being aged $\geqslant 90$ years (p-value: 0.003 , OR: 0.23 ), length of hospital stay (p-value: 0.003, OR: 1.04 ), and preoperative serum albumin level (p-value: 0.037, OR: 0.6). The other evaluated risk factors were not independently found to be associated with all early mortality time.

Conclusion: Patients at or over 90 years old were at risk for all early mortality time points. NLR which is a new and cheap biomarker can be used as a prognostic risk factor for 90-day mortality. The variable of PLR was not found valuable for early mortality.

Categories: Orthopedics, Trauma

Keywords: early mortality, hip fracture, platelet/lymphocyte ratio, neutrophil/lymphocyte ratio, nonagenarian

\section{Introduction}

Hip fractures in elderly patients are a significant public health problem and mainly affect the elderly. It is strongly associated with disability and mortality, and it is well-known that mortality is increased after hip fracture, especially in the first postoperative year [1].

Identifying prognostic factors for mortality in patients with hip fracture can lead to proper management, which is essential if mortality is to be reduced. Several studies have been conducted to investigate mortality rates and the causative factors that lead to death [2,3]. One-year mortality rates vary among the studies, and they suggest that the survival rate is around 64-90\% [4,5]. Both male and female patients after hip fractures have a higher mortality risk than the general population of similar age [6]. The many prognostic factors that the literature proposes may be related to death after hip fracture include age, male gender, comorbidities, American Society of Anesthesiologists (ASA) score, preinjury physical condition, cognitive status, type of fracture, type of surgery, time of delay to surgery, length of hospital stay, and albumin level [7,8]. Besides these standard factors, neutrophil-to-lymphocyte ratio (NLR) and platelet-to-lymphocyte ratio (PLR) were also newly defined parameters to predict mortality after hip fractures $[9,10]$. However, using all these standard and newly defined parameters to predict early mortality is still controversial, and no consensus has yet been reached. This study aimed to identify the standard and newly defined prognostic factors that could predict early mortality after treatment for hip fractures in elderly patients.

\section{Materials And Methods}

With the approval of the local ethics committee, the medical records for 481 patients aged $\geqslant 70$ years who were admitted to our hospital between May 2017 and May 2019 due to low-energy femoral neck or intertrochanteric fractures were reviewed. All patients had been treated with hemiarthroplasty (cemented or 
uncemented) surgery or proximal femoral nailing surgery. All patients were followed up for at least one year after admission or until death. Exclusion criteria included having a pathologic fracture, a concomitant fracture, a periprosthetic fracture, high energy trauma, death before surgery, or having conservative treatment due to a high death risk with surgery. The patients who had data missing for variables other than laboratory data were excluded. The patients who were treated with total hip arthroplasty, proximal femoral plating, or osteosynthesis with dynamic hip screw were also excluded due to the insufficient number to provide reliable data. After applying these criteria, this observational study was performed using the records of 335 patients.

All data were collected from the hospital database. The outcome of interest was mortality, and survival data were obtained from the National Death Registry System in May 2020, which was the endpoint of the study. If the patient was already dead, the time of death was determined as the number of days from admission date until death.

\section{Determination of prognostic factors}

The variables were selected according to their significance as indicated in previously published articles. Patients' sociodemographic data (age, gender), presence of comorbidities, type of fracture (according to Arbeitsgemeinschaft für Osteosynthesefragen $\{\mathrm{AO}\}$ classification), implant type, American Society of Anesthesiologists (ASA) score, type of anesthesia, presence of delirium, time delay to surgery, length of hospital stay, length of stay in intensive care unit (ICU), units of erythrocyte transfusion, and laboratory data (preoperative hemoglobin, platelet count, neutrophil count, total leucocyte count, lymphocyte count, percentage of hemoglobin fall in the first postoperative day, preoperative albumin, blood urea nitrogen \{BUN\}, INR levels and newly defined neutrophil/lymphocyte ratio $\{$ NLR\}, platelet/lymphocyte ratio $\{$ PLR $\}$ parameters) were all assessed in relation to mortality at 30 days, 90 days, or one year.

The neutrophil-to-lymphocyte ratio (NLR) is a newly defined variable and is calculated as the neutrophil count divided by the lymphocyte count. The platelet-to-lymphocyte ratio (PLR) is defined as the platelet count divided by the lymphocyte count.

Age groups were defined as aged $70-79,80-89$, and $\geqslant 90$ years. The type of fracture was classified as either femoral neck or intertrochanteric. Intertrochanteric fractures were further classified as stable or unstable, according to AO classification. The intertrochanteric fractures that belonged to the classes 31A1.1, 31A1.2, 31A1.3, 31A2.1 were accepted as stable, and those in other classes were accepted as unstable. Three types of surgery (cemented hemiarthroplasty, uncemented hemiarthroplasty, and proximal femoral nailing) were included in this study. Regional and general anesthesia were compared as types of anesthesia. Time delay to surgery was defined as the number of days between admission and surgery, and patients were grouped into either within two days or after two days. Comorbidities were collected from patients' International Statistical Classification of Diseases (ICD)-10 codes or from preoperative consultations with anesthesiologists as recorded in the hospital database. Asthma, chronic obstructive pulmonary disease, ischemic heart disease, chronic anemia, hypertension, arrhythmia, Parkinson's disease, dementia, diabetes mellitus, thyroid pathology, peripheral vascular disease, and renal disease were the primary comorbidities. The comorbidity count was determined as having either $0-2$ of them or $\geqslant 3$.

\section{Statistical analysis}

Statistical analysis was carried out using Number Cruncher Statistical System (NCSS) 2007 software (Kaysville, UT: NCSS, LLC.). Descriptive statistical methods were used for means, standard deviation (SD), and percentages. Distribution of variables was determined by the Shapiro-Wilk normality test. All variables were tested by logistic regression analyses to investigate whether they affected 30-day mortality, 90-day mortality, or one-year mortality. Independent t-test was used for comparison of binary groups of variables showing normal distribution, and the Mann-Whitney $\mathrm{U}$ test was used if variables did not show a normal distribution. The chi-square test was used for the comparison of qualitative data. The statistically significant level was set at 0.05 .

\section{Results}

Among the 335 hip fracture patients, 199 (59.4\%) were female and 136 (40.2\%) were male. The mean age of the patients was 83.21 years ( $S D \pm 11.9$, range: $70-105$ years) with $13.7 \%$ of them aged $\geqslant 90$ years. Of the 335 patients, 36 (10.4\%) were deceased within the first 30 days, 72 (21.5\%) of patients within 90 days, and 113 (33.7\%) of them were deceased by the end of one year.

Hip fractures were grouped into intertrochanteric fractures (254 patients, 75.8\%) and neck fractures (81 patients, $24.2 \%$ ). Intertrochanteric fractures were classified as either stable (123 patients, $48.6 \%$ ) or unstable (130 patients, 51.4\%). One intertrochanteric fracture was not classified due to the poor image quality. Two hundred and sixty (77.6\%) patients were treated with hemiarthroplasty (179 intertrochanteric fracture patients, 81 neck fracture patients) and 75 (22.4\%) patients were treated with proximal femoral nailing (all of them were intertrochanteric fracture). Of the hemiarthroplasty group, 40 (15.4\%) patients were cemented and 220 (84.6\%) patients were uncemented. 
on with general anesthesia. Presence of delirium was observed in 51 (16.2\%) patients. Intensive care was required by 184 (54.9\%) patients. Two hundred and sixty-six (79.4\%) patients were operated on within two days, the other 69 (20.6\%) patients were operated on after a two-day period. The presence of $\geqslant 3$

comorbidities was observed in 134 patients (40.1\%). The baseline characteristics of the patients assessed are shown in Table 1.

\begin{tabular}{|c|c|c|c|c|c|}
\hline Factors & $\mathbf{N}$ & Minimum & Maximum & Mean & SD \\
\hline Age & 335 & 70 & 105 & 83.21 & 6.10 \\
\hline Erythrocyte transfusions (unit) & 335 & 0 & 16 & 1.76 & 1.71 \\
\hline Length of hospital stay (days) & 335 & 1 & $1 / 8$ & 12.03 & 15.08 \\
\hline Length of stay in ICU (days) & 184 & 1 & 125 & 6.66 & 14.38 \\
\hline Delay to surgery (days) & 335 & 0 & 7 & 1.58 & 1.36 \\
\hline Total leucocyte count & 334 & 4.02 & 22.08 & 10.37 & 3.31 \\
\hline Preoperative hemoglobin (g/dl) & 334 & 4.00 & 16.80 & 12.01 & 1.19 \\
\hline Postoperative hemoglobin (g/dl) & 327 & 5.30 & 15.50 & 10.33 & 1.47 \\
\hline Postoperative hemoglobin fall (\%) & 327 & -175.00 & 53.10 & 12.42 & 17.63 \\
\hline Platelet count $\left(10^{3} / \mu \mathrm{l}\right)$ & 334 & 71 & 806 & 239.56 & 86.67 \\
\hline Neutrophil count $\left(10^{3} / \mu \mathrm{l}\right)$ & 334 & 0.04 & 19.24 & 8.23 & 3.38 \\
\hline Lymphocyte count $\left(10^{3} / \mu \mathrm{l}\right)$ & 334 & 0.35 & 8.10 & 1.2837 & 0.81 \\
\hline Platelet/lymphocyte ratio (PLR) & 333 & 45.19 & 948.24 & 237.34 & 143.30 \\
\hline Neutrophil/lymphocyte ratıo (NLR) & 334 & 0.03 & 60.00 & 8.8 & 6.59 \\
\hline INR & 327 & 0.86 & 7.79 & 1.12 & 0.48299 \\
\hline Preoperative albumin (g/dl) & 276 & 1.70 & 4.60 & 3.49 & 0.54556 \\
\hline BUN (mg/dl) & 334 & 9.00 & 98.00 & 25.99 & 12.01 \\
\hline
\end{tabular}

\section{TABLE 1: Descriptive statistics of the patients}

SD: standard deviation; BUN: blood urea nitrogen; INR: international normalized ratio

\section{Thirty-day mortality}

Univariate analysis showed that age, age $\geqslant 90$ years, ASA score of 4, preoperative low albumin level, admission to ICU, and length of stay in ICU significantly increased the 30-day mortality rate (Table 2). Subsequently, logistic regression analysis was performed on the significant variables obtained from univariate analysis to determine 30-day mortality. Age $\geqslant 90$ years (p-value: 0.013 , odds ratio \{OR\}: 0.13 ) and ASA score of 4 (p-value: 0.019, OR: 0.22), independently, both had a significant impact on 30-day mortality (Table 3). All the other variables were not significantly associated with 30-day mortality.

\begin{tabular}{|c|c|c|c|c|c|c|c|c|c|c|c|c|c|c|c|c|}
\hline \multirow{2}{*}{\multicolumn{2}{|c|}{ Factors }} & \multicolumn{4}{|c|}{ 30-day mortality } & \multirow{3}{*}{ p-Value } & \multicolumn{4}{|c|}{ 90-day mortality } & \multirow{3}{*}{ p-Value } & \multicolumn{4}{|c|}{ One-year mortality } & \multirow{2}{*}{ p-Value } \\
\hline & & \multicolumn{2}{|c|}{ Alive, n: 300} & \multicolumn{2}{|c|}{ Dead, n: 35} & & \multicolumn{2}{|c|}{ Alive, n: 263} & \multicolumn{2}{|c|}{ Dead, n: 72} & & \multicolumn{2}{|c|}{ Alive, n: 222} & \multicolumn{2}{|c|}{ Dead, n: 113} & \\
\hline \multirow{3}{*}{ Implant type } & Hemiarthroplasty & 230 & $76.67 \%$ & 30 & $85.71 \%$ & & 196 & $74.52 \%$ & 64 & $88.89 \%$ & & 165 & $74.32 \%$ & 95 & $84.07 \%$ & \multirow{3}{*}{$0.043^{*}$} \\
\hline & $\begin{array}{l}\text { Proximal femoral } \\
\text { nailing }\end{array}$ & 70 & $23.33 \%$ & 5 & $14.29 \%$ & \multirow[t]{2}{*}{$0.224^{*}$} & 67 & $25.48 \%$ & 8 & $11.11 \%$ & \multirow[t]{2}{*}{$0.01^{*}$} & 57 & $25.68 \%$ & 18 & $15.93 \%$ & \\
\hline & $\begin{array}{l}\text { Uncemented } \\
\text { hemiarthroplasty }\end{array}$ & 196 & $65.33 \%$ & 24 & $68.57 \%$ & & 166 & $63.12 \%$ & 54 & $75.00 \%$ & & 139 & $62.61 \%$ & 81 & $71.68 \%$ & \\
\hline Implant type & $\begin{array}{l}\text { Cemented } \\
\text { hemiarthroplasty }\end{array}$ & 34 & $11.33 \%$ & 6 & $17.14 \%$ & $0.353^{*}$ & 30 & $11.41 \%$ & 10 & $13.89 \%$ & $0.035^{*}$ & 26 & $11.71 \%$ & 14 & $12.39 \%$ & $0.126^{*}$ \\
\hline
\end{tabular}




\begin{tabular}{|c|c|c|c|c|c|c|c|c|c|c|c|c|c|c|c|c|}
\hline & $\begin{array}{l}\text { Proximal femoral } \\
\text { nailing }\end{array}$ & 70 & $23.33 \%$ & 5 & $14.29 \%$ & & 67 & $25.48 \%$ & 8 & $11.11 \%$ & & 57 & $25.68 \%$ & 18 & $15.93 \%$ & \\
\hline \multirow{2}{*}{ Cement } & Absent & 196 & $85.22 \%$ & 24 & $80.00 \%$ & \multirow{2}{*}{$0.456^{*}$} & 166 & $84.69 \%$ & 54 & $84.38 \%$ & \multirow{2}{*}{$0.951^{*}$} & 139 & $84.24 \%$ & 81 & $85.26 \%$ & \multirow{2}{*}{$0.826^{*}$} \\
\hline & Present & 34 & $14.78 \%$ & 6 & $20.00 \%$ & & 30 & $15.31 \%$ & 10 & $15.63 \%$ & & 26 & $15.76 \%$ & 14 & $14.74 \%$ & \\
\hline \multicolumn{2}{|l|}{ Age } & \multicolumn{2}{|c|}{$82.72 \pm 5.9$} & \multicolumn{2}{|c|}{$87.49 \pm 6.24$} & 0.0001 & \multicolumn{2}{|c|}{$82.39 \pm 6.04$} & \multicolumn{2}{|c|}{$86.24 \pm 5.37$} & $0.0001^{* *}$ & \multicolumn{2}{|c|}{$82.02 \pm 6.1$} & \multicolumn{2}{|c|}{$85.56 \pm 5.42$} & $0.0001^{* \star}$ \\
\hline \multirow{3}{*}{ Age } & $70-79$ & 82 & $27.33 \%$ & 3 & $8.57 \%$ & \multirow{3}{*}{$0.0001^{*}$} & 78 & $29.66 \%$ & 7 & $9.72 \%$ & & 71 & $31.98 \%$ & 14 & $12.39 \%$ & \multirow{3}{*}{$0.0001^{*}$} \\
\hline & $80-89$ & 184 & $61.33 \%$ & 20 & $57.14 \%$ & & 158 & $60.08 \%$ & 46 & $63.89 \%$ & $0.0001^{*}$ & 130 & $58.56 \%$ & 74 & $65.49 \%$ & \\
\hline & $\geq 90$ & 34 & $11.33 \%$ & 12 & $34.29 \%$ & & 27 & $10.27 \%$ & 19 & $26.39 \%$ & & 21 & $9.46 \%$ & 25 & $22.12 \%$ & \\
\hline \multirow{2}{*}{ Gender } & Female & 182 & $60.67 \%$ & 17 & $48.57 \%$ & \multirow{2}{*}{$0.357^{*}$} & 162 & $61.60 \%$ & 37 & $51.39 \%$ & \multirow{2}{*}{$0.240^{*}$} & 142 & $63.96 \%$ & 57 & $50.44 \%$ & \multirow{2}{*}{$0.017^{*}$} \\
\hline & Male & 118 & $39.33 \%$ & 18 & $51.43 \%$ & & 101 & $38.40 \%$ & 35 & $48.61 \%$ & & 80 & $36.04 \%$ & 56 & $49.56 \%$ & \\
\hline \multirow{4}{*}{ ASA score } & 1 & 3 & $1.01 \%$ & 0 & $0.00 \%$ & \multirow{4}{*}{$0.001^{*}$} & 3 & $1.15 \%$ & 0 & $0.00 \%$ & \multirow{4}{*}{$0.023^{*}$} & 3 & $1.36 \%$ & 0 & $0.00 \%$ & \multirow{4}{*}{$0.0001^{*}$} \\
\hline & 2 & 120 & $40.27 \%$ & 11 & $31.43 \%$ & & 109 & $41.76 \%$ & 22 & $30.56 \%$ & & 101 & $45.91 \%$ & 30 & $26.55 \%$ & \\
\hline & 3 & 167 & $56.04 \%$ & 18 & $51.43 \%$ & & 142 & $54.41 \%$ & 43 & $59.72 \%$ & & 110 & $50.00 \%$ & 75 & $66.37 \%$ & \\
\hline & 4 & 8 & $2.68 \%$ & 6 & $17.14 \%$ & & 7 & $2.68 \%$ & 7 & $9.72 \%$ & & 6 & $2.73 \%$ & 8 & $7.08 \%$ & \\
\hline \multirow[t]{2}{*}{ Comorbidities } & $\begin{array}{l}0-2 \\
\text { comorbidities }\end{array}$ & 182 & $60.87 \%$ & 18 & $51.43 \%$ & $0.281^{*}$ & 163 & $62.21 \%$ & 37 & $51.39 \%$ & $0.097^{*}$ & 143 & $64.41 \%$ & 57 & $50.89 \%$ & $0.017^{*}$ \\
\hline & $\geq 3$ comorbidities & 117 & $39.13 \%$ & 17 & $48.57 \%$ & & 99 & $37.79 \%$ & 35 & $48.61 \%$ & & 79 & $35.59 \%$ & 55 & $49.11 \%$ & \\
\hline Delirium & Absent & 253 & $84.33 \%$ & 31 & $88.57 \%$ & $0.509^{*}$ & 227 & $86.31 \%$ & 57 & $79.17 \%$ & $0.135^{*}$ & 195 & $87.84 \%$ & 89 & $78.76 \%$ & $0.029^{*}$ \\
\hline & Present & 47 & $15.67 \%$ & 4 & $11.43 \%$ & & 36 & $13.69 \%$ & 15 & $20.83 \%$ & & 27 & $12.16 \%$ & 24 & $21.24 \%$ & \\
\hline Type of & Spinal & 257 & $85.67 \%$ & 27 & $77.14 \%$ & $0.184^{*}$ & 229 & $87.07 \%$ & 55 & $76.39 \%$ & $0.025^{\star}$ & 193 & $86.94 \%$ & 91 & $80.53 \%$ & $0.123^{*}$ \\
\hline anesthesia & General & 43 & $14.33 \%$ & 8 & $22.86 \%$ & & 34 & $12.93 \%$ & 17 & $23.61 \%$ & & 29 & $13.06 \%$ & 22 & $19.47 \%$ & \\
\hline Erythrocyte tra & insfusion (unit) & $1.74 \pm$ & $=1.70$ & 1.91 & \pm 1.84 & $0.823^{\star \star \star}$ & $1.63 \pm$ & \pm 1.56 & 2.22 & \pm 2.12 & $0.095^{\star * \star}$ & $1.58 \pm$ & \pm 1.29 & 2.11 & $1 \pm 2.29$ & $0.172^{\star * *}$ \\
\hline Length of hosp & ital stay (days) & 12.35 & \pm 15.76 & 9.26 & \pm 6.50 & $0.274^{\star \star \star}$ & 11.17 & $7 \pm 14.99$ & 15.1 & $5 \pm 15.15$ & $0.084^{\star * \star}$ & $9.67 \pm$ & \pm 7.20 & 16.6 & $66 \pm 23.32$ & $0.015^{\star \star *}$ \\
\hline Length of stay & in ICU (days) & $6.64 \pm$ & $=15.42$ & 6.77 & \pm 7.25 & $0.014^{\star * *}$ & $5.01 \pm$ & \pm 14.02 & 10.6 & $5 \pm 14.61$ & $0.001^{* * *}$ & $3.01 \pm$ & \pm 3.15 & 12.2 & $22 \pm 21.43$ & $0.0001^{\text {** }}$ \\
\hline Admission to & Admission (-) & 146 & $48.67 \%$ & 5 & $14.29 \%$ & $0.0001^{*}$ & 133 & $50.57 \%$ & 18 & $25.00 \%$ & $0.0001^{*}$ & 111 & $50.00 \%$ & 40 & $35.40 \%$ & $0.011^{*}$ \\
\hline ICU & Admission (+) & 154 & $51.33 \%$ & 30 & $85.71 \%$ & & 130 & $49.43 \%$ & 54 & $75.00 \%$ & & 111 & $50.00 \%$ & 73 & $64.60 \%$ & \\
\hline Delay to surge & & $1.6 \pm 1$ & .36 & 1.43 & \pm 1.42 & $0.361^{* \star *}$ & $1.56 \pm$ & \pm 1.33 & 1.65 & \pm 1.49 & $0.898^{\star \star *}$ & $1.55 \pm$ & \pm 1.29 & 1.65 & $5 \pm 1.51$ & $0.921^{* * *}$ \\
\hline Delay to & $\leq 2$ days & 238 & $79.33 \%$ & 28 & $80.00 \%$ & $0926^{*}$ & 210 & $79.85 \%$ & 56 & $77.78 \%$ & $0701^{*}$ & 178 & $80.18 \%$ & 88 & $77.88 \%$ & $0622 *$ \\
\hline surgery & $>2$ days & 62 & $20.67 \%$ & 7 & $20.00 \%$ & & 53 & $20.15 \%$ & 16 & $22.22 \%$ & & 44 & $19.82 \%$ & 25 & $22.12 \%$ & \\
\hline Total leucocyte & count $\left(10^{\wedge} 3 / \mu \mathrm{l}\right)$ & $10.3 \pm$ & $=3.19$ & 10.9 & $8 \pm 4.25$ & 0.25 & 10.26 & $6 \pm 3.25$ & 10.7 & $9 \pm 3.56$ & $0.224^{* *}$ & 10.31 & $1 \pm 3.27$ & 10.5 & $50 \pm 3.42$ & $0.627^{\star \star}$ \\
\hline Preoperative $\mathrm{h}$ & emoglobin (g/dl) & 11.99 & \pm 1.78 & 12.2 & $4 \pm 1.92$ & 0.448 & 12.05 & $5 \pm 1.74$ & 11.9 & \pm 1.99 & $0.524^{* *}$ & 12.03 & $3 \pm 1.70$ & 12.0 & $00 \pm 1.96$ & $0.894^{* *}$ \\
\hline Postoperative & hemoglobin (g/dl) & 10.29 & \pm 1.45 & 10.7 & $1 \pm 1.66$ & 0.122 & 10.31 & $1 \pm 1.48$ & 10.4 & $4 \pm 1.48$ & $0.502^{\star \star}$ & 10.29 & $9 \pm 1.44$ & 10.4 & $42 \pm 1.56$ & $0.452^{* *}$ \\
\hline $\begin{array}{l}\text { Percentage of } \\
(\%)\end{array}$ & hemoglobin fall & 12.55 & \pm 18.1 & 11.4 & $2 \pm 13.12$ & $0.276^{\star \star \star}$ & $12.9 \pm$ & \pm 18.03 & 10.6 & $7 \pm 16.09$ & $0.274^{\star \star *}$ & 12.77 & $7 \pm 18.74$ & 11.7 & $77 \pm 15.28$ & $0.352^{* * *}$ \\
\hline $\begin{array}{l}\text { Preoperative } p \\
\left(10^{3} / \mu \mathrm{l}\right)\end{array}$ & latelet count & 240.6 & $7 \pm 87.11$ & 230. & $09 \pm 83.49$ & $0.495^{* *}$ & 240.0 & $06 \pm 85.08$ & 237. & $75 \pm 92.85$ & $0.842^{* *}$ & 238.6 & $66 \pm 87.96$ & 241 & $.33 \pm 84.48$ & $0.791^{\text {** }}$ \\
\hline $\begin{array}{l}\text { Preoperative n } \\
\left(10^{3} / \mu \mathrm{l}\right)\end{array}$ & eutrophil count & $8.14 \pm$ & $=3.28$ & 8.99 & \pm 4.19 & $0.161^{* *}$ & $8.09 \pm$ & \pm 3.34 & 8.76 & \pm 3.52 & $0.139^{\star *}$ & $8.15 \pm$ & \pm 3.42 & 8.39 & $9 \pm 3.34$ & $0.553^{* *}$ \\
\hline $\begin{array}{l}\text { Preoperative ly } \\
\left(10^{3} / \mu \mathrm{l}\right)\end{array}$ & /mphocyte count & $1.30 \pm$ & $=0.84$ & 1.14 & \pm 0.50 & $0.282^{* *}$ & $1.3 \pm 0$ & & 1.22 & \pm 0.82 & $0.431^{* *}$ & $1.29 \pm$ & \pm 0.72 & 1.28 & $8 \pm 0.98$ & $0.952^{* *}$ \\
\hline Platelet/lymphc & ocyte ratio (PLR) & 236.1 & $1 \pm 141.18$ & 247. & $85 \pm 162.19$ & $0.771^{\star * \star}$ & 232.5 & $58 \pm 133.72$ & 254. & $93 \pm 174.16$ & $0.562^{\star \star *}$ & 231.0 & $07 \pm 132.11$ & 249 & $.74 \pm 163.1$ & $0.568^{\star *}$ \\
\hline $\begin{array}{l}\text { Neutrophil/lym } \\
\text { (NLR) }\end{array}$ & phocyte ratio & $8.70 \pm$ & $=6.62$ & 9.78 & \pm 6.40 & $0.244^{\star * *}$ & $8.36 \pm$ & \pm 5.89 & 10.4 & $5 \pm 8.54$ & $0.042^{\star \star *}$ & $8.52 \pm$ & \pm 6.10 & 9.38 & $8 \pm 7.46$ & $0.303^{* * *}$ \\
\hline
\end{tabular}




\section{Cureus}

\begin{tabular}{|c|c|c|c|c|c|c|c|c|c|c|c|c|c|c|c|c|}
\hline \multicolumn{2}{|c|}{ Preoperative INR } & \multicolumn{2}{|c|}{$1.12 \pm 0.51$} & \multicolumn{2}{|c|}{$1.11 \pm 0.13$} & $0.877^{\star \star}$ & \multicolumn{2}{|c|}{$1.10 \pm 0.33$} & \multicolumn{2}{|c|}{$1.21 \pm 0.83$} & $0.077^{* \star}$ & \multicolumn{2}{|c|}{$1.10 \pm 0.36$} & \multicolumn{2}{|c|}{$1.16 \pm 0.66$} & $0.275^{\star \star}$ \\
\hline \multicolumn{2}{|c|}{ Preoperative albumin (g/dl) } & \multicolumn{2}{|c|}{$3.53 \pm 0.52$} & \multicolumn{2}{|c|}{$3.23 \pm 0.63$} & $0.003^{\star \star}$ & \multicolumn{2}{|c|}{$3.54 \pm 0.51$} & \multicolumn{2}{|c|}{$3.37 \pm 0.62$} & $0.023^{\star *}$ & \multicolumn{2}{|c|}{$3.57 \pm 0.52$} & \multicolumn{2}{|c|}{$3.37 \pm 0.57$} & $0.003^{\star \star}$ \\
\hline \multicolumn{2}{|c|}{ Preoperative BUN (mg/dl) } & \multicolumn{2}{|c|}{$25.63 \pm 11.98$} & \multicolumn{2}{|c|}{$29.17 \pm 11.99$} & $0.099^{\star *}$ & \multicolumn{2}{|c|}{$24.97 \pm 11.26$} & \multicolumn{2}{|c|}{$29.75 \pm 13.89$} & $0.003^{\star *}$ & \multicolumn{2}{|c|}{$24.8 \pm 10.57$} & \multicolumn{2}{|c|}{$28.34 \pm 14.19$} & $0.011^{\star \star}$ \\
\hline \multirow{2}{*}{$\begin{array}{l}\text { Type of } \\
\text { fracture }\end{array}$} & Trochanteric & 227 & $75.67 \%$ & 27 & $77.14 \%$ & \multirow{2}{*}{$0.847^{*}$} & 199 & $75.67 \%$ & 55 & $76.39 \%$ & \multirow{2}{*}{$0.899^{*}$} & 170 & $76.58 \%$ & 84 & $74.34 \%$ & \multirow{2}{*}{$0.651^{*}$} \\
\hline & Neck & 73 & $24.33 \%$ & 8 & $22.86 \%$ & & 64 & $24.33 \%$ & 17 & $23.61 \%$ & & 52 & $23.42 \%$ & 29 & $25.66 \%$ & \\
\hline \multirow{2}{*}{$\begin{array}{l}\text { AO } \\
\text { classification }\end{array}$} & Stable & 114 & $50.44 \%$ & 9 & $33.33 \%$ & \multirow{2}{*}{$0.093^{*}$} & 100 & $50.51 \%$ & 23 & $41.82 \%$ & \multirow{2}{*}{$0.254^{*}$} & 84 & $49.41 \%$ & 39 & $46.99 \%$ & \multirow{2}{*}{$0.717^{*}$} \\
\hline & Unstable & 112 & $49.56 \%$ & 18 & $66.67 \%$ & & 98 & $49.49 \%$ & 32 & $58.18 \%$ & & 86 & $50.59 \%$ & 44 & $53.01 \%$ & \\
\hline
\end{tabular}

\section{TABLE 2: Univariate analysis of 30-day mortality, 90-day mortality, one-year mortality}

${ }^{*}$ Chi-square test.

**Independent t-test.

***Mann-Whitney U test.

ASA: American Society of Anesthesiologists; ICU: intensive care unit; NLR: neutrophil/lymphocyte ratio; BUN: blood urea nitrogen; INR: international normalized ratio

\begin{tabular}{|c|c|c|c|c|c|c|c|}
\hline \multirow{2}{*}{ Factors } & & \multirow{2}{*}{$\beta$-Value } & \multirow{2}{*}{ p-Value } & & \multirow{2}{*}{ OR } & \multicolumn{2}{|c|}{$95 \% \mathrm{Cl}$ for OR } \\
\hline & & & & & & Lower & Upper \\
\hline \multirow{2}{*}{ Age (years) } & $80-89$ & 0.74 & 0.117 & \multirow{2}{*}{0.038} & 0.48 & 0.19 & 1.20 \\
\hline & $\geq 90$ & 2.07 & 0.013 & & 0.13 & 0.03 & 0.65 \\
\hline \multirow{3}{*}{ ASA score } & ASA 2 & 1.44 & 0.998 & & - & - & - \\
\hline & ASA 3 & 1.30 & 0.061 & 0.140 & 0.27 & 0.07 & 1.06 \\
\hline & ASA 4 & 1.52 & 0.019 & & 0.22 & 0.06 & 0.78 \\
\hline Admission to ICU & & 1.08 & 0.063 & 0.063 & 0.34 & 0.11 & 1.06 \\
\hline Albumin (g/dl) & & -0.55 & 0.148 & 0.148 & 0.58 & 0.27 & 1.22 \\
\hline
\end{tabular}

\section{TABLE 3: Logistic regression analysis for 30-day mortality}

ASA: American Society of Anesthesiologists; ICU: intensive care unit; OR: odds ratio; Cl: confidence interval

\section{Ninety-day mortality}

In univariate analysis, age, age $\geqslant 90$ years, ASA score of 4 , and general anesthesia were found to be predictors of 90 -day mortality (p-value: $0.0001,0.0001,0.023$, and 0.025 , respectively). Proximal femoral nailing technique was a significant predictor for survival (p-value: 0.035). Admission to ICU and length of stay in ICU were both found to be predictors of 90-day mortality (p-value: 0.0001 and 0.001 , respectively). Preoperative low albumin, high BUN levels, and the newly defined high NLR as laboratory variables were all significant for 90-day mortality (p-value: $0.023,0.003$, and 0.042 , respectively) (Table 2).

Following logistic regression analysis, age (p-value: 0.002), age of 80-89 years (p-value: 0.032, OR: 0.43), age $\geqslant 90$ years (p-value: 0.001 , OR: 0.13 ), general anesthesia (p-value: 0.016 , OR: 0.41 ), high NLR (p-value: 0.028, OR: 1.05), and high BUN levels (p-value: 0.049, OR: 1.02) were all significant predictors for 90-day mortality (Table 4 ). 


\section{Cureus}

\begin{tabular}{|c|c|c|c|c|c|c|c|}
\hline \multirow{2}{*}{ Factors } & & \multirow{2}{*}{$\beta$-Value } & \multirow{2}{*}{ p-Value } & & \multirow{2}{*}{ OR } & \multicolumn{2}{|c|}{$95 \% \mathrm{Cl}$ for OR } \\
\hline & & & & & & Lower & Upper \\
\hline Hemiarthroplasty & & 0.76 & 0.093 & & 2.15 & 0.88 & 5.24 \\
\hline \multirow{2}{*}{ Age (years) } & $80-89$ & 0.85 & 0.032 & \multirow{2}{*}{0.002} & 0.43 & 0.20 & 0.93 \\
\hline & $\geq 90$ age & 2.02 & 0.001 & & 0.13 & 0.04 & 0.41 \\
\hline \multirow{3}{*}{ ASA score } & ASA 2 & 1.21 & 0.998 & \multirow{3}{*}{0.467} & 0.00 & 0.00 & - \\
\hline & ASA 3 & 1.02 & 0.116 & & 0.36 & 0.10 & 1.29 \\
\hline & ASA 4 & 0.92 & 0.136 & & 0.40 & 0.12 & 1.34 \\
\hline \multicolumn{2}{|l|}{ General anesthesia } & 0.88 & 0.016 & & 0.41 & 0.20 & 0.85 \\
\hline \multicolumn{2}{|l|}{ Admission to ICU } & 0.43 & 0.238 & & 0.65 & 0.32 & 1.33 \\
\hline \multicolumn{2}{|l|}{ NLR } & 0.05 & 0.028 & & 1.05 & 1.01 & 1.09 \\
\hline \multicolumn{2}{|l|}{ Albumin (g/dl) } & -0.21 & 0.369 & & 0.16 & 0.42 & 1.38 \\
\hline \multicolumn{2}{|l|}{ BUN (mg/dl) } & 0.02 & 0.049 & & 1.02 & 1.00 & 1.05 \\
\hline
\end{tabular}

\section{TABLE 4: Logistic regression analysis for 90-day mortality}

ASA: American Society of Anesthesiologists; ICU: intensive care unit; NLR: neutrophil/lymphocyte ratio; BUN: blood urea nitrogen; OR: odds ratio; CI: confidence interval

\section{One-year mortality}

Age (p-value: 0.0001$)$, age $\geqslant 90$ years ( $p$-value: 0.0001$)$, male ( $p$-value: 0.017$)$, hemiarthroplasty surgery ( $p$ value: 0.043 ), presence of delirium ( $p$-value: 0.029 ), presence of $\geqslant 3$ comorbidities ( $p$-value: 0.017 ), ASA score of 4 (p-value: 0.0001 ), length of hospital stay (p-value: 0.015 ), admission to ICU (p-value: 0.011), length of stay in ICU (p-value: 0.0001), preoperative low albumin (p-value: 0.003), and high BUN levels (p-value: 0.011 ) all reached statistical significance for one-year mortality in the univariate analysis conducted (Table 2).

In the subsequent logistic regression analysis, predictor factors for one-year mortality were determined as age $\geqslant 90$ years ( $p$-value: 0.003 ), length of hospital stay ( $p$-value: 0.003 ), and preoperative low albumin levels (p-value: 0.037) (Table 5). 


\section{Cureus}

\begin{tabular}{|c|c|c|c|c|c|c|c|}
\hline \multirow{2}{*}{ Factors } & & \multirow{2}{*}{$\beta$-Value } & \multirow{2}{*}{\multicolumn{2}{|c|}{$\mathrm{p}$-Value }} & \multirow{2}{*}{ OR } & \multicolumn{2}{|c|}{$95 \% \mathrm{Cl}$ for OR } \\
\hline & & & & & & Lower & Upper \\
\hline Hemiarthroplasty & & 0.58 & 0.125 & & 1.79 & 0.85 & 3.77 \\
\hline \multirow{2}{*}{ Age (years) } & $80-89$ & 0.47 & 0.225 & \multirow{2}{*}{0.006} & 0.62 & - & - \\
\hline & $>90$ & 1.46 & 0.003 & & 0.23 & 0.09 & 0.60 \\
\hline Gender & Male & 0.42 & 0.134 & & 0.66 & 0.38 & 1.14 \\
\hline \multirow{3}{*}{ ASA score } & ASA 2 & 0.21 & 0.999 & & - & - & - \\
\hline & ASA 3 & 0.87 & 0.170 & 0.443 & 0.42 & 0.12 & 1.45 \\
\hline & ASA 4 & 0.46 & 0.443 & & 0.63 & 0.20 & 2.05 \\
\hline Comorbidities (>3) & & 0.44 & 0.135 & & 0.64 & 0.36 & 1.15 \\
\hline Presence of delirium & & 0.21 & 0.557 & & 0.81 & 0.40 & 1.64 \\
\hline Length of hospital stay (days) & & 0.04 & 0.003 & & 1.04 & 1.01 & 1.06 \\
\hline Admission to ICU & & 0.14 & 0.665 & & 1.14 & 0.62 & 2.10 \\
\hline Albumin (g/dl) & & -0.52 & 0.037 & & 0.60 & 0.37 & 0.97 \\
\hline BUN (mg/dl) & & 0.01 & 0.798 & & 1.00 & 0.98 & 1.03 \\
\hline
\end{tabular}

TABLE 5: Logistic regression analysis for one-year mortality

ASA: American Society of Anesthesiologists; ICU: intensive care unit; BUN: blood urea nitrogen; OR: odds ratio; CI: confidence interval

\section{Discussion}

In this study, which aimed to determine the prognostic factors for mortality in hip fracture patients, being aged $\geqslant 90$ years and having an ASA score of 4 were found to be associated with 30-day mortality; age, being aged 80-89 years, being aged $\geqslant 90$ years, general anesthesia, high NLR and BUN levels were all associated with 90-day mortality, while being aged $\geqslant 90$ years, length of hospital stay, and preoperative albumin levels were associated with one-year mortality. Being aged 90 years or older was the only significant prognostic factor that affected all early mortality timings. Being a nonagenarian seems to be one of the strongest indicators for mortality $[11,12]$. Nonagenarians have more comorbidities than the younger elderly, and their functional levels and mobility are far more limited. The population of the world is aging, and the number of people who will reach their 90 s is increasing. Therefore, fragility fractures of the hip will inevitably increase in the coming years. Reducing comorbidities, presenting a better public health service, and a routine followup for osteoporosis in the elderly will provide the opportunity to lower both early and total mortality rates.

NLR is an inflammatory biomarker and has been proposed for use in some fields of medicine, such as gastrointestinal disease, oncology, and cardiology [13-15]. NLR was first used in hip fracture patients by Forget et al. [16]. They stated that admission NLR was not useful as a predictor for mortality and proposed NLR on the fifth postoperative day. In contrast, Fisher et al. showed that admission NLR was a significant independent risk factor in geriatric hip fractures for postoperative myocardial injury, high inflammatory response, and in-hospital death [9]. PLR is another proposed inflammatory biomarker, similar to NLR, and it has been studied in relation to adverse cardiac outcomes and oncology [17,18]. PLR was first investigated as a prognostic risk factor in hip fractures by Emektar et al. [10]. Although they initially stated that high PLR at admission was found to be predictive of one-year mortality, they concluded that it was clinically insignificant. In this study, we found that NLR was an independent prognostic risk factor, in both univariate and logistic regression analysis, for 90-day mortality, but it did not emerge as a significant prognostic factor for one-year mortality. PLR evaluation was consistent with the literature and was not found to be significant as a prognostic factor [10]. This study showed that the male gender had a significantly higher mortality rate in univariate analysis of 90-day mortality, but when logistic regression analysis was applied, it was not found to be as significant as previous studies had concluded [1]. In comparison, Kannegaard et al. and Endo et al. showed that male patients had an excess mortality rate compared to women $[19,20]$. In the present study, the patients were evaluated preoperatively with an ASA score, and an ASA score of 4 was associated with 30-day mortality in both the univariate and logistic regression analysis. Although an ASA score of 4 was also associated with 90-day mortality and one-year mortality, it did not remain significant after logistic regression analyses. In some studies, ASA score has been shown to be a prognostic factor for early mortality [21]. Other studies have denied that there is a correlation between mortality and ASA scores for patients over 90 years [22]. It is a well-accepted fact that increased comorbidities bring higher mortality rates. Although the presence of $\geqslant 3$ comorbidities was significant for one-year mortality in the univariate 
analysis, this parameter did not remain significant. Due to the retrospective nature of this study, the comorbidities of the patients could not be perfectly determined.

The effect of anesthetic technique on early mortality has been researched in numerous studies, and the majority of them demonstrate that general anesthesia is a negative prognostic factor [23]. In contrast, a Cochrane review in 2016 showed that type of anesthesia had no effect on one-year mortality [24]. In another review, Van Waesberghe et al. investigated whether type of anesthesia affected 30-day mortality, and no significant difference was found [25]. In our study, general anesthesia was associated with higher 90-day mortality rate after applying logistic regression analysis. We found no relation with 30-day or one-year mortality, in agreement with the literature.

The mortality rate did not differ with the type of fracture (neck vs intertrochanteric, stable intertrochanteric vs unstable intertrochanteric). Although hemiarthroplasty surgery was associated with 90-day and one-year mortality, after logistic regression analysis, it was not significant. Neither cemented nor uncemented hemiarthroplasty was superior to the other. The literature is controversial regarding the type of fracture and the type of implant [7].

Hip fracture patients are at risk for confusion and delirium due to trauma. Dolan et al. found admission delirium to be an important factor for functionality and poor outcomes [26]. They stated that it did not remain as a significant predictor for mortality after adjustment for confounding variables. However, opposite results have also been indicated [27]. In the present study, delirium was associated with 90-day mortality in the univariate analysis but was not associated with mortality after logistic regression analysis.

A longer in-hospital stay was significantly associated with one-year mortality. We determined length of hospital stay as a significant prognostic factor for one-year mortality. Nikkel et al. stated that increased length of stay resulted in an increased mortality rate in a US cohort [28]. The findings of our study and those of Nikkel et al. contrast sharply with a Swedish study by Nordström et al., in which shortened hospital stay was found to be a risk factor for mortality [29].

Preoperative serum albumin level, which identifies the nutritional status of patients, is one of the most researched variables for early mortality. Studies show that hypoalbuminemia $(\leqslant 3.5 \mathrm{~g} / \mathrm{dl})$ is associated with one-year mortality [30]. Our data confirmed this, and it was determined as a risk factor for one-year mortality in our study. Chronic renal failure is thought to be a prognostic risk factor for early mortality. Although high BUN levels, which are an indicator of chronic renal failure, were associated with 90-day mortality in this study, it should not be considered a valuable or reliable prognostic factor due to its susceptibility to diet and muscle loss. We found no significant relation between early mortality and the variables of delay before surgery, the number of erythrocyte transfusions, preoperative hemoglobin, percentage of hemoglobin fall, platelet count, lymphocyte count, total leucocyte count, neutrophil count, or preoperative INR.

This study has a few limitations. It was not possible to obtain some of the laboratory data or the preoperative mobility status of the patients due to the retrospective nature of the study. The importance of preoperative and postoperative mobilization levels of the patients is known for postoperative mortality and morbidity. Because of the retrospective course of our study, we could not exactly evaluate the mobilization level. Other treatment modalities, such as total hip arthroplasty, osteosynthesis with proximal femoral plating, or dynamic hip screw were also excluded due to the lesser number of patients operated with these treatment methods. Numerous factors for mortality have been proposed, but we were only able to investigate the most important of these factors. This is another limitation of the this study.

\section{Conclusions}

In conclusion, a detailed assessment of risk factors is crucial in order to reduce the mortality rate after hip fractures. Our data suggest that being aged $\geqslant 90$ years, having an ASA score of 4 , general anesthesia, high NLR ratio, high BUN levels, longer length of hospital stay, and preoperative low albumin levels are independent risk factors for early mortality. We suggest that NLR, which is a new and cheap prognostic biomarker, should continue to be researched extensively in prospective studies with larger sample sizes.

\section{Additional Information \\ Disclosures}

Human subjects: Consent was obtained or waived by all participants in this study. Süleyman Demirel University Medicine Faculty Clinical Research Ethical Committee issued approval \#72867572.050.01.0464356. Animal subjects: All authors have confirmed that this study did not involve animal subjects or tissue. Conflicts of interest: In compliance with the ICMJE uniform disclosure form, all authors declare the following: Payment/services info: All authors have declared that no financial support was received from any organization for the submitted work. Financial relationships: All authors have declared that they have no financial relationships at present or within the previous three years with any organizations that might have an interest in the submitted work. Other relationships: All authors have declared that there are no other relationships or activities that could appear to have influenced the submitted work. 


\section{Acknowledgements}

Data are available on reasonable request. The data are stored as de-identified participant data, which are available on request to the corresponding author.

\section{References}

1. Aharonoff GB, Koval KJ, Skovron ML, Zuckerman JD: Hip fractures in the elderly: predictors of one year mortality. J Orthop Trauma. 1997, 11:162-5. 10.1097/00005131-199704000-00004

2. Boylan MR, Riesgo AM, Paulino CB, Slover JD, Zuckerman JD, Egol KA: Mortality following periprosthetic proximal femoral fractures versus native hip fractures. J Bone Joint Surg Am. 2018, 100:578-85. 10.2106/JBJS.17.00539

3. Frisch NB, Wessell N, Charters M, Greenstein A, Shaw J, Peterson E, Guthrie ST: Hip fracture mortality: differences between intertrochanteric and femoral neck fractures. J Surg Orthop Adv. 2018, 27:64-71. 10.3113/jsoa.2018.0064

4. Roche JJ, Wenn RT, Sahota O, Moran CG: Effect of comorbidities and postoperative complications on mortality after hip fracture in elderly people: prospective observational cohort study. BMJ. 2005, 331:10.1136/bmj.38643.663843.55

5. Karagiannis A, Papakitsou E, Dretakis K, Galanos A, Megas P, Lambiris E, Lyritis GP: Mortality rates of patients with a hip fracture in a southwestern district of Greece: ten-year follow-up with reference to the type of fracture. Calcif Tissue Int. 2006, 78:72-7. 10.1007/s00223-005-0169-6

6. Omsland TK, Emaus N, Tell GS, et al.: Mortality following the first hip fracture in Norwegian women and men (1999-2008). A NOREPOS study. Bone. 2014, 63:81-6. 10.1016/j.bone.2014.02.016

7. Härstedt M, Rogmark C, Sutton R, Melander O, Fedorowski A: Impact of comorbidity on 6-month hospital readmission and mortality after hip fracture surgery. Injury. 2015, 46:713-8. 10.1016/j.injury.2014.12.024

8. Lim KK, Matchar DB, Chong JL, Yeo W, Howe TS, Koh JS: Pre-discharge prognostic factors of physical function among older adults with hip fracture surgery: a systematic review. Osteoporos Int. 2019, 30:92938. 10.1007/s00198-018-04831-5

9. Fisher A, Srikusalanukul W, Fisher L, Smith P: The neutrophil to lymphocyte ratio on admission and shortterm outcomes in orthogeriatric patients. Int J Med Sci. 2016, 13:588-602. 10.7150/ijms.15445

10. Emektar E, Corbacioglu SK, Dagar S, Uzunosmanoglu H, Safak T, Cevik Y: Prognostic value of the neutrophil-lymphocyte and platelet- lymphocyte ratios in predicting one-year mortality in patients with hip fractures and aged over 60 years. Eurasian J Emerg Med. 2017, 16:165-70. 10.5152/eajem.2017.51523

11. Barceló M, Francia E, Romero C, Ruiz D, Casademont J, Torres OH: Hip fractures in the oldest old. Comparative study of centenarians and nonagenarians and mortality risk factors. Injury. 2018, 49:2198-202. 10.1016/j.injury.2018.09.043

12. Kim JW, Kim DH, Jang EC, Lee YK, Koo KH, Ha YC: Mortality and its risk factors in nonagenarians after hip fractures. J Orthop Sci. 2019, 24:850-4. 10.1016/j.jos.2019.02.019

13. Cigsar G, Yildirim AC, Anuk T, et al.: Neutrophil to lymphocyte ratio on appendectomy of geriatric and nongeriatric patients. J Invest Surg. 2017, 30:285-90. 10.1080/08941939.2016.1241324

14. Tan TP, Arekapudi A, Metha J, Prasad A, Venkatraghavan L: Neutrophil-lymphocyte ratio as predictor of mortality and morbidity in cardiovascular surgery: a systematic review. ANZ J Surg. 2015, 85:414-9. 10.1111/ans.13036

15. Guo J, Chen S, Chen Y, Li S, Xu D: Combination of CRP and NLR: a better predictor of postoperative survival in patients with gastric cancer. Cancer Manag Res. 2018, 10:315-21. 10.2147/CMAR.S156071

16. Forget P, Moreau N, Engel H, Cornu O, Boland B, De Kock M, Yombi JC: The neutrophil-to-lymphocyte ratio (NLR) after surgery for hip fracture (HF). Arch Gerontol Geriatr. 2015, 60:366-71.

10.1016/j.archger.2014.11.008

17. Kwon HC, Kim SH, Oh SY, et al.: Clinical significance of preoperative neutrophil-lymphocyte versus platelet-lymphocyte ratio in patients with operable colorectal cancer. Biomarkers. 2012, 17:216-22. 10.3109/1354750X.2012.656705

18. Gürsoy OM, Karakoyun S, Kalçik M, et al.: Usefulness of novel hematologic inflammatory parameters to predict prosthetic mitral valve thrombosis. Am J Cardiol. 2014, 113:860-4. 10.1016/j.amjcard.2013.11.029

19. Kannegaard PN, van der Mark S, Eiken P, Abrahamsen B: Excess mortality in men compared with women following a hip fracture. National analysis of comedications, comorbidity and survival. Age Ageing. 2010, 39:203-9. 10.1093/ageing/afp221

20. Endo Y, Aharonoff GB, Zuckerman JD, Egol KA, Koval KJ: Gender differences in patients with hip fracture: a greater risk of morbidity and mortality in men. J Orthop Trauma. 2005, 19:29-35. 10.1097/00005131200501000-00006

21. Chatterton BD, Moores TS, Ahmad S, Cattell A, Roberts PJ: Cause of death and factors associated with early in-hospital mortality after hip fracture. Bone Joint J. 2015, 97-B:246-51. 10.1302/0301-620X.97B2.35248

22. Ishida Y, Kawai S, Taguchi T: Factors affecting ambulatory status and survival of patients 90 years and older with hip fractures. Clin Orthop Relat Res. 2005, 208-15. 10.1097/01.blo.0000159156.40002.30

23. Beaupre LA, Jones CA, Saunders LD, Johnston DW, Buckingham J, Majumdar SR: Best practices for elderly hip fracture patients. A systematic overview of the evidence. J Gen Intern Med. 2005, 20:1019-25. 10.1111/j.1525-1497.2005.00219.x

24. Guay J, Parker MJ, Gajendragadkar PR, Kopp S: Anaesthesia for hip fracture surgery in adults . Cochrane Database Syst Rev. 2016, 2:10.1002/14651858.CD000521.pub3

25. Van Waesberghe J, Stevanovic A, Rossaint R, Coburn M: General vs. neuraxial anaesthesia in hip fracture patients: a systematic review and meta-analysis. BMC Anesthesiol. 2017, 17:87. 10.1186/s12871-017-0380-9

26. Dolan MM, Hawkes WG, Zimmerman SI, Morrison RS, Gruber-Baldini AL, Hebel JR, Magaziner J: Delirium on hospital admission in aged hip fracture patients: prediction of mortality and 2-year functional outcomes. J Gerontol A Biol Sci Med Sci. 2000, 55:M527-34. 10.1093/gerona/55.9.m527

27. Ruggiero C, Bonamassa L, Pelini L, et al.: Early post-surgical cognitive dysfunction is a risk factor for mortality among hip fracture hospitalized older persons. Osteoporos Int. 2017, 28:667-75. 10.1007/s00198016-3784-3 


\section{Cureus}

28. Nikkel LE, Kates SL, Schreck M, Maceroli M, Mahmood B, Elfar JC: Length of hospital stay after hip fracture and risk of early mortality after discharge in New York state: retrospective cohort study. BMJ. 2015,

351:10.1136/bmj.h6246

29. Nordström P, Michaëlsson K, Hommel A, Norrman PO, Thorngren KG, Nordström A: Geriatric rehabilitation and discharge location after hip fracture in relation to the risks of death and readmission. J Am Med Dir Assoc. 2016, 17:10.1016/j.jamda.2015.07.004

30. Silva TJ, Jerussalmy CS, Farfel JM, Curiati JA, Jacob-Filho W: Predictors of in-hospital mortality among older patients. Clinics (Sao Paulo). 2009, 64:613-8. 10.1590/S1807-59322009000700002 